\title{
EXPERIMENTAL EVIDENCE OF A HARD TRANSITION TO CHAOS
}

\author{
E. DEL RÍO, J. R. SANMARTÍN and O. LÓPEZ-REBOLLAL \\ E. T. S. Ingenieros Aeronáuticos, Universidad Politécnica, \\ 28040 Madrid, Spain
}

Received August 18, 1997; Revised April 8, 1998

\begin{abstract}
A generic, sudden transition to chaos has been experimentally verified using electronic circuits. The particular system studied involves the near resonance of two coupled oscillators at $2: 1$ frequency ratio when the damping of the first oscillator becomes negative. We identified in the experiment all types of orbits described by theory. We also found that a theoretical, 1D limit map fits closely a map of the experimental attractor which, however, could be strongly disturbed by noise. In particular, we found noisy periodic orbits, in good agreement with noise theory.
\end{abstract}

\section{Introduction}

A hard in amplitude transition to chaos in a class of dissipative flows of broad physical applicability was recently reported [López-Rebollal \& Sanmartín, 1995; Hughes \& Proctor, 1990; Sanmartín et al., 1993]. These flows exhibited a state of rest for negative values of a parameter $\Gamma$, and a fully developed chaotic attractor for $\Gamma$ positive, no matter how small. The $\Gamma \rightarrow+0$ limit of long-time solutions was a singular solution: It did not exist at $\Gamma=0$, relating to the fact that the limits $t \rightarrow+\infty$ and $\Gamma \rightarrow+0$ do not commute (the singular solution takes an infinite time to make a single leg on the attractor). This led to exactly $1 \mathrm{D}$, noninvertible maps, with vanishing Cantor structures and to chaotic dynamics with vanishing Lyapunov exponents. The transition has been recently shown to be structurally stable [López-Rebollal et al., 1998].

A particular system exhibiting this transition (2:1 near-resonance of waves or oscillators with quadratic coupling) was analyzed in detail by López-Rebollal and Sanmartín [1995]. In the present paper we carry out an experimental test of the theory for this case. To model the system we constructed two coupled electronic oscillators, using a technique which reported very good results to us in dealing with nonlinear, in particular chaotic, problems [del Río et al., 1992, 1994]. We paid special attention to the effects of noise, which should be high in our system, as discussed in numerical calculations [Hughes \& Proctor, 1990].

Section 2 briefly reviews the main results of the theory for the case of $2: 1$ resonance. Model circuit and experimental procedure are described in Sec. 3. Experimental results and a comparison to theory are presented in Sec. 4 . In Sec. 5 we consider circuit-noise effects on the system. We summarize results in Sec. 6.

\section{Summarized Theory of the Transition}

The simplest model for the hard transition is the weakly nonlinear, conservative coupling of two harmonic oscillators [López-Rebollal \& Sanmartín, 1995],

$$
\begin{aligned}
\ddot{x}- & 2 \Gamma \dot{x}+4 \omega_{0}^{2} x \\
& =-\frac{1}{2 m}\left(V_{x x x}^{0} x^{2}+2 V_{x x y}^{0} x y+V_{x y y}^{0} y^{2}\right),
\end{aligned}
$$




$$
\begin{aligned}
& \ddot{y}+2 \gamma \dot{y}+\omega_{0}^{2} y \\
& =-\nu \omega_{0} y-\frac{1}{2 m}\left(V_{x x y}^{0} x^{2}+2 V_{x y y}^{0} x y+V_{y y y}^{0} y^{2}\right),
\end{aligned}
$$

at frequencies $2 \omega_{0}$ and $\sqrt{\omega_{0}^{2}+\nu \omega_{0}}$ (with $\nu / \omega_{0}$ small), when the linear damping for $x$ changes sign; we expanded the two-dimensional (2D) potential energy $V(x, y)$, with $V_{x x x}^{0}$ representing $\partial_{V}^{3} / \partial x^{3}$ at the origin (a stable equilibrium point), etc. Writing

$$
\begin{aligned}
& x=\frac{4 m \omega_{0}}{V_{x y y}^{0}} a_{1} \cos \left(2 \omega_{0} t+\psi\right), \\
& y=\frac{8 m \omega_{0}}{V_{x y y}^{0}} a_{2} \cos \left(\omega_{0} t+\theta\right)
\end{aligned}
$$

and averaging over the $2 \omega_{0}$ and $\omega_{0}$ periods, one finds that small dampings $-2 \Gamma$ and $2 \gamma>0$, as well as resonant coupling terms and frequency mismatch $\nu>0$, make the normalized amplitudes and phase constants for the respective oscillations, slowly evolve according to equations [López-Rebollal \& Sanmartín, 1995]

$$
\dot{a}_{1}=\Gamma a_{1}-a_{2}^{2} \sin \beta,
$$

$$
\begin{aligned}
\dot{a}_{2} & =-\gamma a_{2}+a_{1} a_{2} \sin \beta, \\
\dot{\beta} & =\nu+2 a_{1} \cos \beta-\frac{a_{2}^{2}}{a_{1}} \cos \beta,
\end{aligned}
$$

with $\beta \equiv 2 \theta-\psi$. This 3D flow has been extensively studied as a model for the ubiquitous three-wave, quadratic interaction, with one wave instable and the two other waves equally damped [Pikovsky \& Rabinovich, 1981; Wersinger et al., 1980; Meunier et al., 1982].

Systems 3(a)-3(c) has an invariant surface, $\Sigma\left(a_{2}=0\right)$. The state of rest $\left(a_{1}=a_{2}=0\right)$ is a global attractor for $\Gamma<0$ and is unstable for $\Gamma>0$; there is then a fixed point $P\left[a_{2 P}{ }^{2} \sin ^{2} \beta_{P}=\Gamma \gamma\right.$, $\left.a_{1 P} \sin \beta_{P}=\gamma, \tan \beta_{P}=(\Gamma-2 \gamma) / \nu\right]$. For $\Gamma=0$ there is a second invariant surface, $\Sigma^{\prime}(\nu+$ $2 a_{1} \cos \beta=0$ ), which meets $\Sigma$ at a line of fixed points $\Lambda$ (Fig. 1); point $P$ now lies on $\Lambda$. The surface $\Sigma^{\prime}$ is made of heteroclinic orbits and nested on $P$, joining $\Lambda$-points pairwise (see orbits I, II or III in Fig. 1). Also, $a_{1}$ is constant along $\Sigma$-orbits, which are heteroclinic above the bottom $Q$ of $\Lambda(m \rightarrow \bar{m}$ in Fig. 1) and periodic below $Q$. The $\Gamma=0$ attractor is the union of the periodic orbits below $Q$, and the arc $P Q$ of $\Lambda$ [López-Rebollal \& Sanmartín, 1995].

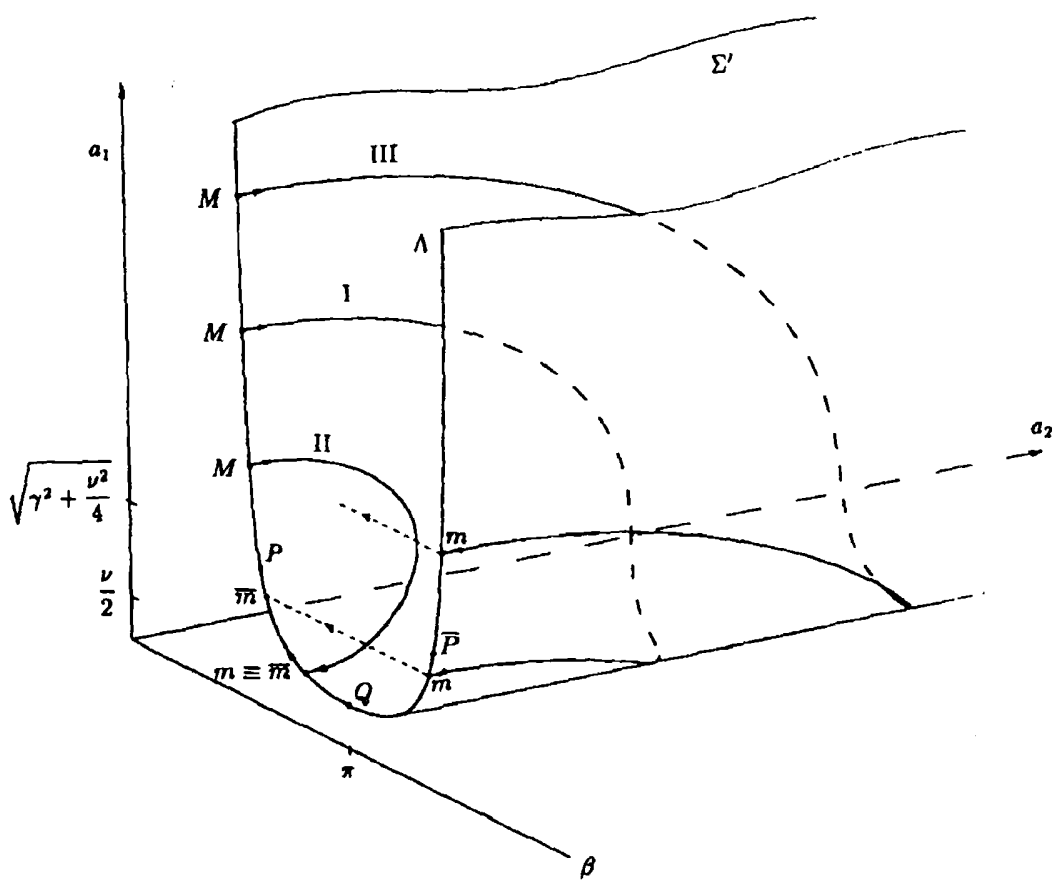

Fig. 1. Heteroclinic orbits for Eqs. (3a)-(3c), with $\Gamma$ vanishing and $\nu / \gamma$ low enough. Invariant surfaces $a_{2}=0(\Sigma)$ and $\nu+2 a_{1} \cos \beta=0\left(\Sigma^{\prime}\right)$ intersect at a line of fixed points $\Lambda_{;} P$ is a fixed point existing at $\Gamma>0$ too, $Q$ the bottom of $\Lambda, \bar{P}$ the symmetric of $P$ on $\Lambda$. For $\Gamma \rightarrow+0$, orbits rise at a vanishing rate from $\bar{m}$ below $P$, to $M^{\prime}$ above $P$. 
The entire heteroclinic structure disappears with $\Sigma^{\prime}$ and $\Lambda$ at positive $\Gamma$. A ghost of the $\Gamma=0$ invariant structure, however, allows the analytical determination of an exactly 1D, first return map for $a_{1}$-maxima on the chaotic attractor, in the limit $\Gamma \rightarrow+0$. For very small $\Gamma$ and $\nu / \gamma<2, P$ is a saddle-focus with a $2 \mathrm{D}$ unstable manifold near $\Sigma$; as $\Gamma \rightarrow+0$ the spiralling motion lying between $P$ and $\Sigma$ is crashed onto $\Lambda$, making points on this line drift along it at a vanishing rate. In the $\left\{a_{1}, a_{2}, \beta\right\}$ space there are three types of "complete" limit orbits, taking $a_{1}$ from a maximum $a_{1 M}$ to the next one $a_{1 M^{\prime}}$; this determines the map of maxima.

Type I: These complete orbits consist of three steps:

(1) A $\Gamma=0$ heteroclinic orbit on the surface $\Sigma^{\prime}$, from a point $M$ above $P$ on the $\beta<\pi$ branch of line $\Lambda$, to a point $m$ lying on the opposite branch, between $Q$ and $\bar{P}$ (symmetric of $P$ on $\Lambda$, Fig. 1).

(2) A $\Gamma=0$, heteroclinic orbit on $\Sigma$, from $m$ to its symmetric $\bar{m}$ between $Q$ and $P$.

(3) A vanishingly slow $(\Delta t \sim 1 / \Gamma)$ rise along $\Lambda$, for $\Gamma \rightarrow+0$, from $\bar{m}$ to a new maximum $M^{\prime}$ above $P$. Note that $a_{2}$ vanishes in the last two steps.

Type II: For $M$ low enough above $P, m$ itself lies between $Q$ and $P$, and the second step of Type I orbits is missing.

Type III: For $M$ high enough, $m$ lying above $P$, there is no third step.

The resulting 1D map is bimodal with the two critical points corresponding to $m$ at $\bar{P}$ or $Q$. This exact limit map, which exhibits a chaotic attractor at low enough $\nu / \gamma$, has the form [López-Rebollal \& Sanmartín, 1995; Hughes \& Proctor, 1990]

$$
\begin{gathered}
F\left(Z_{M^{\prime}}\right)=F\left(\left|Z_{M}-2\right|\right) \quad \text { if } \quad Z_{M} \leq 3 \\
Z_{M^{\prime}}=\left|Z_{M}-2\right| \quad \text { if } \quad Z_{M} \geq 3
\end{gathered}
$$

$$
\begin{aligned}
F\left(Z, \frac{\nu}{\gamma}\right) & \equiv 2 Z-\ln \left(\frac{\nu^{2}}{4 \gamma^{2}}+Z^{2}\right)-\frac{\nu}{\gamma} \tan ^{-1}\left(\frac{2 \gamma}{\nu} Z\right) \\
Z & =\left(\frac{a_{1}^{2}}{\gamma^{2}}-\frac{\nu^{2}}{4 \gamma^{2}}\right)^{\frac{1}{2}}
\end{aligned}
$$

\section{Experimental Model}

We constructed an (analog) model circuit for Eqs. (1a) and (1b). Figure 2 shows both, a block diagram for the circuit, and the connection to the

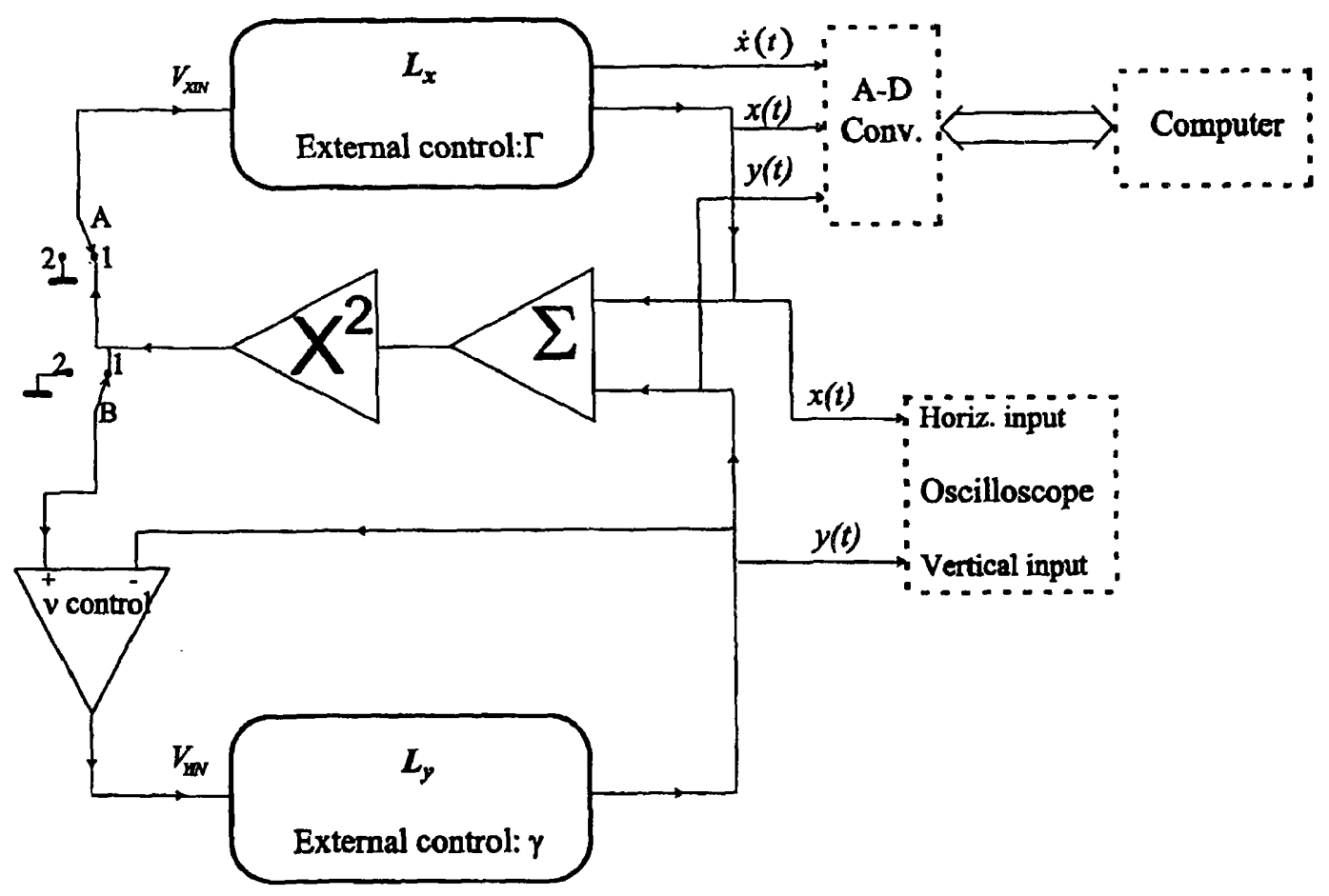

Fig. 2. Block diagram for circuit and data acquisition device. 
data acquisition device. There are two similar linear oscillators in the circuit (blocks $L_{x}$ and $L_{y}$ in the figure), tuned at $2 \omega_{0}$ for the $x$ variable, and near $\omega_{0}$ for $y$. The output voltage of each oscillator is used as input in an adding amplifier, and the signal is then applied to the input of a four-quadrant analog multiplier BB4206. Instead of integrators we used for the blocks $L_{x}$ and $L_{y}$ the circuit given by del Río et al. [1992], which requires just one operational amplifier per linear oscillator, with feedback close to unity, thus reducing internal and external fluctuations and ensuring a fairly linear amplifier in a wide frequency range (note that circuit noise can affect the dynamics as later seen in Sec. 5). Also, one may easily proceed continuously from negative to positive values of the damping factor [del Río et al., 1992], as required in the experiment.

For data acquisition we used a programmable 12 bit analog-digital converter under computer control. This system makes possible to measure successive maxima of $x(t)$ in Fig. $2\{\dot{x}(t)=0$ and $\ddot{x}(t)<0\}$, using the following procedure: Setting the trigger threshold at $0 \mathrm{~V}$, we use the signal $\dot{x}(t)$ as trigger channel, choosing the low to high transition as trigger mode. For each transition we make 20 samples in the $x(t)$ channel, each delayed $1 / 20 \mathrm{~ms}$, and covering $1 \mathrm{~ms}$ overall; ten samples are acquired prior to the trigger condition, and ten afterwards. We then fit a parabola to the samples and calculate the maximum, called $x_{n}$; with this method we filter the high frequency noise. Next, following Eqs. (2a) and (2b), we write $x(t)=X(t) \cos \left(2 \omega_{0} t+\psi\right)$, $y(t)=Y(t) \cos \left(\omega_{0} t+\theta\right)$. Since the characteristic time of $X(t)$ will be much larger than $1 / 2 \omega_{0}$, the discrete sequence of values for maxima of $x(t)$, $\left\{x_{n}\right\}$, approximately determines the function $X(t)$, and its maxima $X_{M}$ (proportional to $a_{1 M}$ ), which occur at time intervals close to the period $\pi / \omega_{0}$.

To determine $\omega_{0}$ one sets the switch $B$ in position 2, ensuring that, after a transient time, we will have $y(t)=0$. Under this condition and with $\Gamma$ tuned close to zero, the nonlinear $x$-oscillator will make autonomous oscillations. If the amplitude is small (around $0.05 \mathrm{~V}$ ), the nonlinear term is negligible as compared with the linear term, and the frequency $2 \omega_{0}$ is directly given by a frequency meter connected to the $x(t)$ output signal. The result for our circuit is $\omega_{0}=101.250 \mathrm{rad} / \mathrm{s}$. In a similar way we can measure the frequency of the $y$-oscillator, which gives $\nu$ in Eq. (1b).

To measure negative damping factors, $\Gamma>0$, we start with switch $A$ in position 2 and the autonomous, linear $x$-oscillator amplitude close to zero, and measure the exponential growth of the $x$ amplitude. We measure $\gamma$ in a similar way; since the linear $y$-oscillator is damped, one must start here with a large amplitude, typically $5 \mathrm{~V}$.

\section{Comparison to Theory}

Figure 3(a) shows $x_{n}$ as a function of $n$ for two typical orbits as described in Sec. 2. The range $10<n<290$ will be shown below to correspond to an orbit of Type I in Fig. 1, which consists of three steps. During the third step (the slow rise on $\Lambda$ with $a_{2} \propto Y \approx 10^{-3} \mathrm{~V}$ or less) the oscilloscope (Fig. 2) displays a horizontal segment of increasing length until the system leaves the $Y \approx 0$ surface [at $n \approx 290$ in Fig. 3(a)] as a vertical explosion, which is the first step of the next orbit. To compare

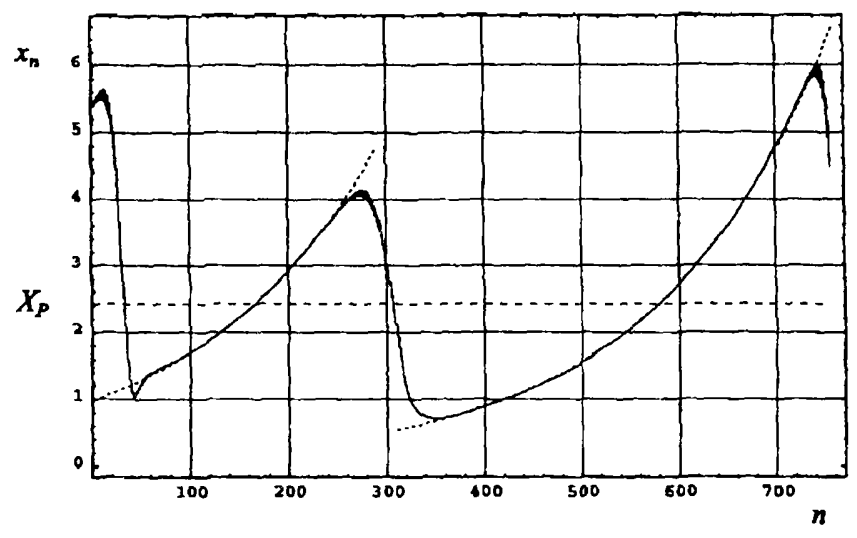

(a)

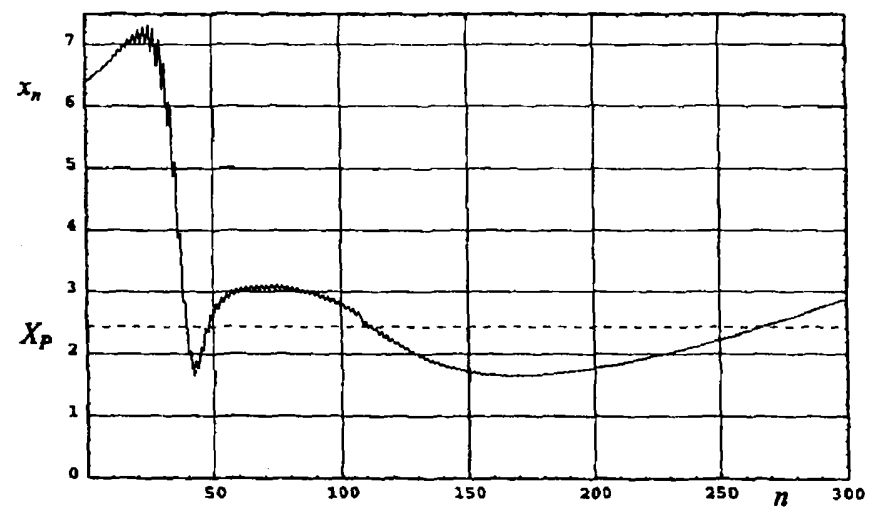

(b)

Fig. 3. Typical $x_{n}$-series (in volts) for $\nu=0.630 \mathrm{rad} / \mathrm{s}$, $\gamma=1.802 \mathrm{rad} / \mathrm{s}$. (a) Types I and II orbits; the two dashed lines are exponential laws fitting the experimental data. (b) Type III orbit. For the differences in profile curvature, see main text. 
the step in Fig. 3(a) with theory, note that for $Y \approx 0$, Eq. (3a) gives $\dot{X}(t) \approx \Gamma X(t)$; the experimental series for $x_{n}$ will then grow exponentially. A linear least-square fit of the $\ln x_{n}$-data determines $\Gamma=0.175 \mathrm{rad} / \mathrm{s}$, in good agreement with the value $\Gamma=0.179 \mathrm{rad} / \mathrm{s}$ that had been obtained from measurements with the $x$-oscillator when uncoupled ( $\sim 2 \%$ of error), as explained in Sec. 3 . The dotted line in Fig. 3(a) for $n<290$ represents the theoretical prediction for $x_{n}$.

Values $n \approx 290$ to $n \approx 720$, following a lower maximum of $x_{n}$ (lower $X_{M}$ ), will be shown to correspond to a Type II orbit in Fig. 1. A similar exponential fit to the data also gives $\Gamma=0.175 \mathrm{rad} / \mathrm{s}$. Again, the dotted line in Fig. 3(a) for $n>310$ is the theoretical prediction for $x_{n}$.

Differences of profile curvature near the respective minima in Fig. 3(a), and before the rise on line $\Lambda$, are in agreement with theory. Note that Eqs. (3a) $-(3 c)$ exactly yield:

$$
\begin{aligned}
\ddot{a}_{1}= & \Gamma^{2} a_{1}+\frac{a_{2}^{4}}{a_{1}} \cos \beta+2 a_{2}^{2}\left[\left(\gamma-\frac{\Gamma}{2}\right) \sin \beta\right. \\
& \left.-a_{1}-\frac{\nu}{2} \cos \beta\right] .
\end{aligned}
$$

Close to the surface $\Sigma$ but before the $\Lambda$-rise we have $\Gamma<<a_{2}<<1$, the bracket term being then dominant in Eq. (6), with the sign of the bracket determining the sign of $\ddot{a}_{1}$. Figure 4 shows both $\Lambda$ and the line $L$ where that bracket vanishes for the $\nu-\gamma$ values of Figs. 3(a) and 3(b), and $\Gamma<<\gamma$. Above and to the right of $L$, the bracket term is negative. Clearly, a Type I orbit, which has $\ddot{a}_{1}>0$ at the $\beta=\pi$ minimum in Fig. 1, should have $\ddot{a}_{1}<0$ when reaching $\Sigma$ at the $\beta>\pi$ branch of $\Lambda$, below $\bar{P}$, and should again have $\ddot{a}_{1}>0$ on the $\beta<\pi$ branch, following the second step. One can see these two signchanges, previous to the $\Lambda$-rise, in the $10<n<290$ orbit of Fig. 3(a).

A Type II orbit, on the other hand, does not cross the $\beta=\pi$ surface, and its minimum occurs near the surface $\Sigma$, where $\ddot{a}_{1}$ is small as seen in Eq. (6); this should result in a broad minimum for $a_{1}$. Also, since the orbit approaches $\Sigma$ near the $\beta<\pi$ branch of $\Lambda$, where $\ddot{a}_{1}$ is positive as at the minimum, it will lack the two sign-changes of a Type I orbit. Again, one can see both features in the $290<n<720$ orbit of Fig. 3(a).

Finally, a Type III orbit does cross the $\beta=\pi$ surface and thus exhibits a narrow minimum, as in Type I; also, since the orbit reaches $\Lambda$ on its

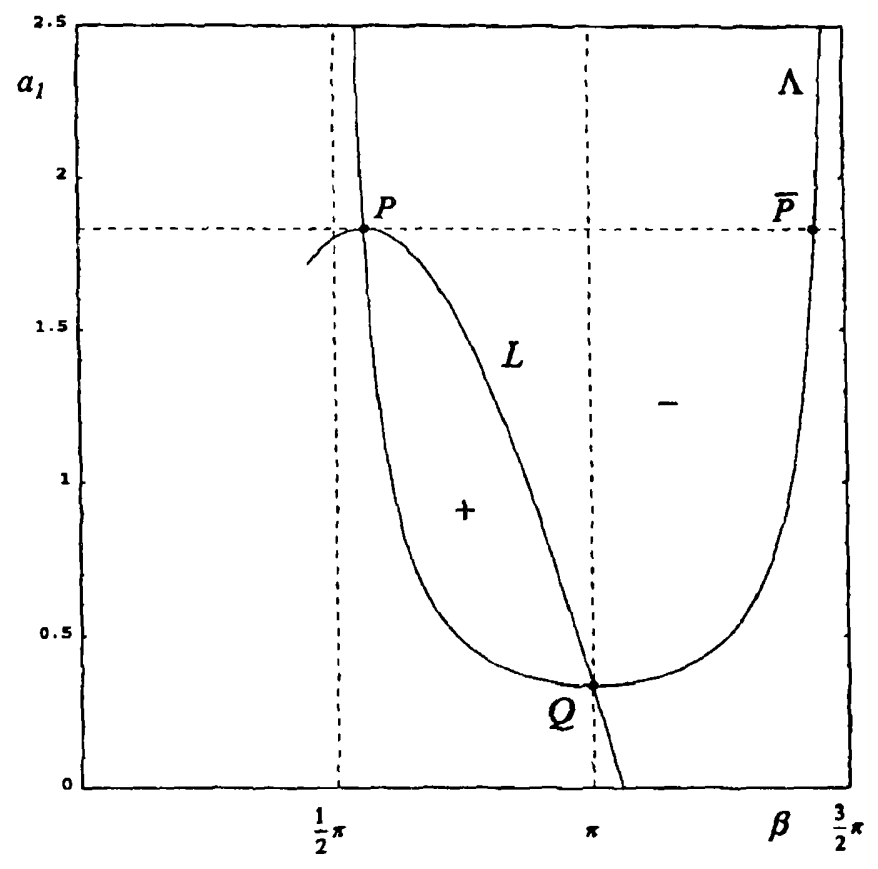

Fig. 4. Plane $a_{1}-\beta$ for the same $\nu, \gamma$ values of Fig. 3 and $\Gamma$ vanishing, showing $\Lambda$, and the line $L\left(0=\gamma \sin \beta-a_{1}-\frac{\nu}{2} \cos \beta\right)$ dividing the plane in two regions according to the $\ddot{x}(t)$ sign as indicated.

$\beta>\pi$ branch (above $\bar{P}$ in Fig. 4), $\ddot{a}_{1}$ should exhibit a first sign-change. On the other hand, the orbit will clearly lack the second sign-change, keeping $\ddot{a}_{1}<0$ while crossing to the $\beta<\pi$ branch. All these fear tures are found in Fig. 3(b) for an orbit starting at a $x_{n}$-maximum higher than those in Fig. 3(a).

Finally, for positive $\Gamma$, no matter how small, the theory predicts a chaotic attractor within certain domain of parameters $\nu, \gamma$, with $X_{M}$ governed by the limit map $(\Gamma \rightarrow+0)$ given by Eqs. (4) and (5). Experimental points in Fig. 5(a) describe a first-return map for $X_{M}$ corresponding to the values of $\nu, \gamma$ and $\Gamma$ of Figs. 3(a) and 3(b). There are 599 maxima in a $x_{n}$-series of $2 \times 10^{5}$ points. Those parts of the complete series represented in Figs. 3(a) and 3(b) include three maxima in the first figure, and two in the second. One may observe in the figures that the $x_{n}$ signal has a trianglewave component with a period twice the period $\pi / \omega_{0}$ of the linear $x$-oscillator. This is a result of nonresonant coupling terms in Eqs. (1a) and (1b) that were not taken into account in Eqs. (3a)-(3c), and which are entirely negligible in the vanishingamplitude limit only; such as effect made necessary to calculate $X_{M}$ as a parabolic least-square fit of a set of points around each $x_{n}$-hill. Also shown in Fig. 5(a) is the theoretical map, Eqs. (4) and (5), 


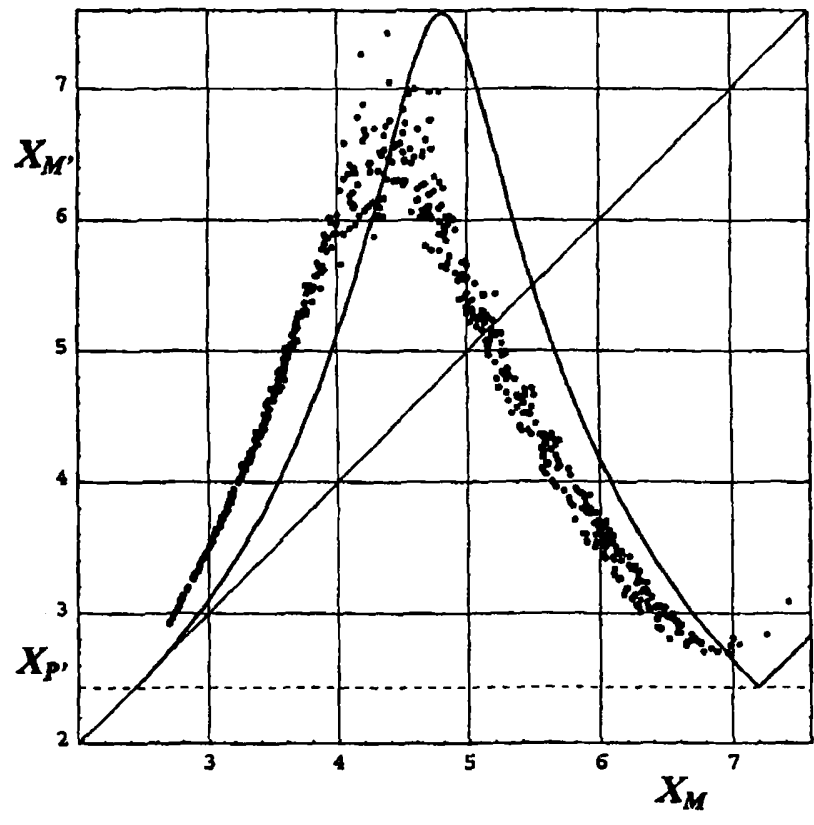

(a)

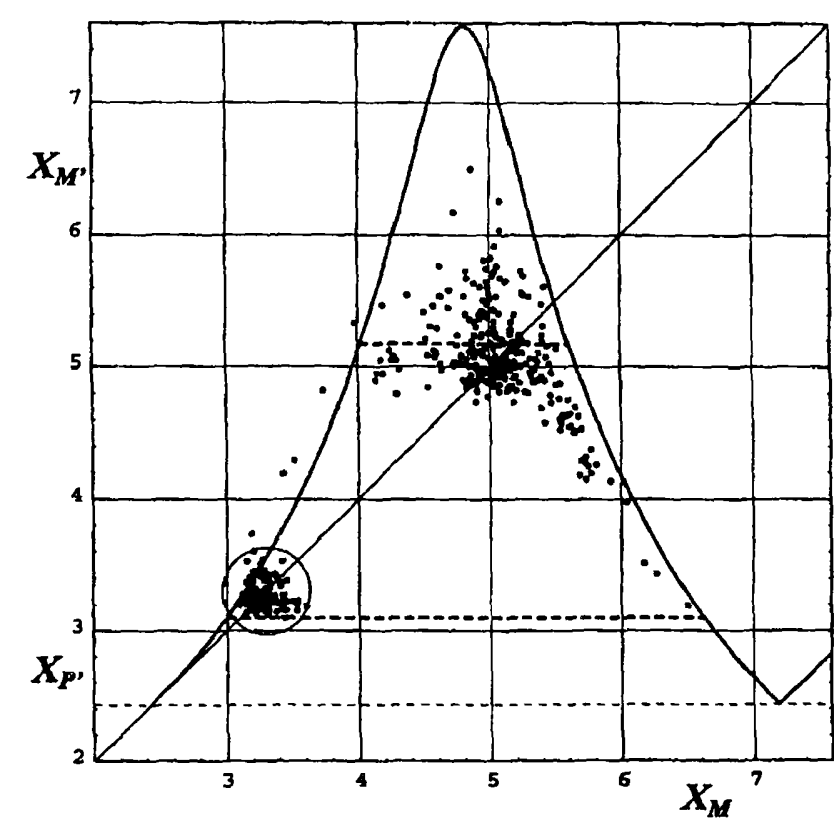

(b)

Fig. 5. Experimental return map maxima of $X_{M}$ (in volt) for $\nu=0.630 \mathrm{rad} / \mathrm{s}, \gamma=1.802 \mathrm{rad} / \mathrm{s}$, as obtained from $2 \times 10^{5} x_{n}$ points. The continuous line represents the corresponding exact, $\Gamma \rightarrow+0$ limit map. (a) $\Gamma=0.179 \mathrm{rad} / \mathrm{s}$. (b) $\Gamma=0.092 \mathrm{rad} / \mathrm{s}$ (for points outside the circle) and $\Gamma=0.013 \mathrm{rad} / \mathrm{s}$ (for points inside the circle). The two upper dashed lines are the corresponding cut-off levels given by Eq. (8).

for $\Gamma \rightarrow+0$ (full line); the agreement with the experimental data is good. The leftward shift of the data from the $\Gamma \rightarrow+0$ map may be shown to be due to the actual value of $\Gamma$ not being small enough (see Fig. 6 in [Sanmartín et al., 1993]). On the other hand, the substantial dispersion of the data, particularly around the top of the map, is a result of the high sensitivity of our system to noise, which is studied in the next section.

\section{Noise Effects at Very Small $\Gamma$}

The rise from $\bar{m}$ to $M^{\prime}$ on $\Lambda$, in Types I and II orbits, is very slow, as determined by $\dot{X}(t) \approx \Gamma X(t)$,

$$
t\left(M^{\prime}\right)-t(\bar{m}) \approx \frac{1}{\Gamma} \ln \frac{X_{M^{\prime}}}{X_{\bar{m}}}=0\left(\frac{1}{\bar{\Gamma}}\right) ;
$$

the times for the partial rises from $\bar{m}$ to $P$, and from $P$ to $M^{\prime}$, are similarly of order $1 / \Gamma$ (Fig. 1 ). Note also that $\dot{Y} / Y\left(\equiv \dot{a}_{2} / a_{2}=-\gamma+a_{1} \sin \beta\right)$ is negative during the long rise $\bar{m} \rightarrow P$, and changes sign at $P ; Y$ will thus have a (very small) minimum value at $P$. That minimum is clearly smaller when the rise to $P$ is longer, i.e. the lower the point $\vec{m}$. This explains why data dispersion is largest near the top of the experimental map in Fig. 5(a). Also, note how the weak dispersion at the map bottom on the right of the maximum is noticeably larger than on the left; this is because Type II orbits (points $X_{M}$ lying in the branch of the map at the left of the maximum) lack the fast step $m \rightarrow \bar{m}$, which contributes somewhat to the $Y$-decrease for Type I orbits (points $X_{M}$ between the maximum of the map and its minimum, at $X_{M} \approx 7 \mathrm{~V}$ ). Finally, the few data for $X_{M}>7 \mathrm{~V}$ correspond to Type III orbits.

The $x$ and $y$ output signals of our circuit have a RMS noise value of $\varepsilon \approx 0.6 \times 10^{-3} \mathrm{~V}$. Since the noise level is constant, one can make it eventually dominant by decreasing the (positive) value of $\Gamma$. Points outside the circle in Fig. 5(b) represent the experimental data for the same $\nu, \gamma$ values of Fig. 5(a), but with $\Gamma=0.092 \mathrm{rad} / \mathrm{s}$. We stored a $x_{n}$-series of $2 \times 10^{5}$ points getting 296 maxima; this is less than the number of maxima for Fig. 5(a) because the characteristic time for orbits is determined by the slow $\Lambda$-rise, which is proportional to $1 / \Gamma[\mathrm{Eq} .(7)]$. The map domain affected by noise is now substantially greater than in Fig. 5(a); on the other hand, points far from that domain are closer to the limit map than in Fig. 5(a), as expected. 
Points inside the circle in Fig. 5(b) correspond to a value $\Gamma=0.013 \mathrm{rad} / \mathrm{s}$. There are 120 maxima here for $2 \times 10^{5}$ cycles of the $x$-oscillator. Orbit dynamics is now entirely dominated by noise.

We may use a crude model of noise for this system [Hughes \& Proctor, 1990, 1992]. In this model, if the minimum $Y_{P}$ in the noiseless system is higher than the noise level $\varepsilon$, one assumes that the flow is unaffected by noise, whereas if $Y_{P}$ is smaller than $\varepsilon$ one sets $Y_{p}=\varepsilon$ before starting the rise from $P$. Using the variable $Z$ defined in Eq. (6), Eq. (4a) becomes [Hughes \& Proctor, 1990]

$$
F\left(Z_{M^{\prime}}\right)=F(1)+2 \frac{\Gamma}{\gamma}\left|\ln \left(\varepsilon^{\prime}\right)\right|
$$

$Z_{M^{\prime}}$ being then independent of the input value $Z_{M}$ $\left(X_{M^{\prime}}\right.$ independent of $\left.X_{M}\right) ; \varepsilon^{\prime}$ is the noise level for $a_{2}$. The value $X_{M^{\prime}}$ given by Eq. (8) is a cutoff level for the noiseless map, so in this approximation the corrected map is determined by choosing for each $X_{M}$ the lower of the two $X_{M^{\prime}}$ values given by Eqs. (4a) and (8). For $\Gamma$ small enough, $0.013 \mathrm{rad} / \mathrm{s}$ for the experimental data inside the circle in Fig. 5(b), the cut-off level $(3.1 \pm 0.3 \mathrm{~V}$, as indicated by the dash line) lies below the fixed point of the noiseless map, so we obtain a noisy periodic orbit. The mean value of the mentioned data is $3.26 \mathrm{~V}$ in very satisfactory agreement with the cut-off level given by Eq. (8). On the other hand, if the cut-off level lies above the fixed point of the map, the map shape remains close to a flattop tent, as for Fig. 5(a) (cut-off at $6.71 \pm 0.08 \mathrm{~V}$ ). The maximum disturbance of the tent-shape corresponds to a cut-off value around the fixed point of the experimental map as for points outside the circle in Fig. 5(b) (5.18 $0.4 \mathrm{~V}$ upper dash line). With this first degree of approximation given by Eq. (8), which does not take the noise-distribution into account, only its level $\varepsilon$, we have no results for the $X_{M}$-distribution.

As we can see, the return time for the map in the absence of noise, $\tau \equiv t\left(M^{\prime}\right)-t(M)$, is dominated by the exponential rise on $\Lambda ; \Gamma \tau$ will thus be independent of $\Gamma$ for each iteration on the $\Gamma \rightarrow+0$ limit map. Plotting the mean value of $\tau, \bar{\tau}$, versus $1 / \Gamma$, at small $\Gamma$ and fixed values of $\nu$ and $\gamma$, one should approximately obtain a straight line through the origin. To calculate $\bar{\tau}$ from the experimental data, we divide the number of points of a large $x_{n}$ series $\left(2 \times 10^{5}\right.$ points in our case) by the corresponding number of $X_{M}$ maxima; this yields $\bar{\tau}$

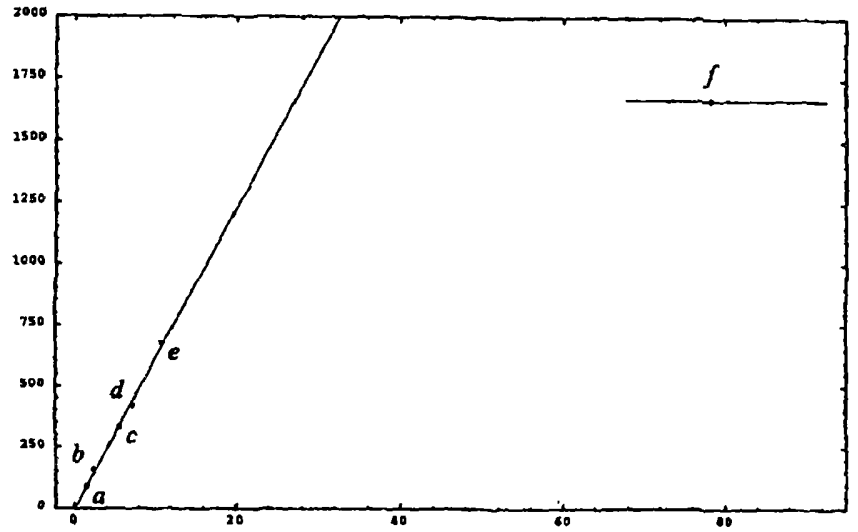

Fig. 6. Experimental mean return-time for the map, $\bar{\tau}$, versus $1 / \Gamma$. The continuos line is obtained by a leastsquare fitting of the five points closest to origin. Point $f$ has a large error bar. Gamma values are: (a) $0.658 \mathrm{rad} / \mathrm{s}$, (b) $0.411 \mathrm{rad} / \mathrm{s}$, (c) $0.179 \mathrm{rad} / \mathrm{s}$, (d) $0.138 \mathrm{rad} / \mathrm{s}$, (e) $0.092 \mathrm{rad} / \mathrm{s}$, and (f) $0.013 \mathrm{rad} / \mathrm{s}$.

in periods of the $x$-oscillator. Results are shown in Fig. 6 for six values of $\Gamma$. The figure also shows the least-square fit line for the five points closest to the origin, in very good agreement with the noiseless prediction. The last point, $f$, which lies well below that line, corresponds to the fully noisy case shown inside the circle in Fig. 5(b); the noise reduces $\bar{\tau}$ by reducing the duration of the rise $P \rightarrow M^{\prime}$. Points $c$ and $e$ correspond to the data in Fig. 5(a) and outside the circle in Fig. 5(b), respectively. Point a corresponds to a value $\Gamma=0.658 \mathrm{rad} / \mathrm{s}$. Note that the noiseless, $\Gamma \rightarrow+0$, law, for $\bar{\tau}(1 / \Gamma)$, (a straight line through the origin) proves to be surprisingly robust: The attractor for case $e$ was seen to be strongly disturbed by noise in Fig. 5(b), while the experimental data for case $a$ lie actually closer to a two fixed-points cycle than to the limit map of Fig. 5(a).

\section{Summary of Results}

We have presented experimental evidence of a hard transition to chaos for $2: 1$ near-resonant coupling of oscillators. The particular system we used are two-coupled, analog, electronic oscillators, designed specifically for this purpose. By checking curvature features and a exponential rise stage in the amplitude versus time profiles, we identified in the experiment the three types of orbits described by theory [López-Rebollal \& Sanmartín, 1995]. We also found that the experimental maxima of the amplitude fit closely the analytical, 1D limit map of Eqs. (4) 
and (5). We have further shown how the experimental attractor can be strongly disturbed by noise in the circuit. In particular, we found the noisy periodic orbit, in good agreement with noise-chaos theory [Hughes \& Proctor, 1990].

\section{Acknowledgments}

This work was supported by DGYCIT (Spain) under grant PB94-0417-C03-01.

\section{References}

del Rio, E., Rodriguez-Lozano, A. \& Velarde, M. G. [1992] "A prototype Helmholtz-Thompson nonlinear oscillator," Rev. Sci. Instrum. 63, 4208-4212.

del Río, E., Rodriguez-Lozano, A. \& Velarde, M. G. [1994] "Melnikov criterion and its experimental test using a nonlinear oscillator," Chaos, Solitons \& Fractals 4, 255-262.

Hughes, D. W. \& Proctor, M. R. E. [1990] "Chaos and the effect of noise in a model of three-wave model coupling," Physica D46, 163-176.

Hughes, D. W. \& Proctor, M. R. E. [1992] "Nonlinear three-wave interaction with non-conservative coupling," J. Fluid Mech. 244, 583-604.

López-Rebollal, O. \& Sanmartín, J. R. [1995] "A generic, hard transition to chaos," Physica D89, 204-221.

López-Rebollal, O., Sanmartín, J. R. \& del Río, E. [1998] "Sudden transition to chaos in plasmas wave interactions," Phys. Plasmas 5, 2861-2867.

Meunier, C., Bussac, M. N., \& Laval, G. [1982] "Intermittency at the onset of stochasticity in nonlinear resonant coupling processes" Physica D4, 236-243.

Pikovsky, A. S. \& Rabinovich, I. [1981] "Stochastic oscillations in dissipative systems," Physica D2, 8-24.

Sanmartín, J. R., López-Rebollal, O. \& dePaola, N. [1993] "Non-periodic driving of coupled oscillators: A spherical swing," Physica D69, 148-162.

Wersinger, J.-M., Finn, J. M., \& Ott, E. [1980] "Bifurcation and 'strange' behaviour in instability saturation by nonlinear three-wave mode coupling," Phys. Fluids 23, 1142-1154. 